\title{
Lire la littérature médiévale en classe de français langue étrangère : une utopie ?
}

\section{Gaëlle Burg}

Si le corpus littéraire traditionnellement utilisé dans l'enseignement secondaire et supérieur du FLE emprunte le plus souvent à la littérature des $19^{e}, 20^{e}$ et $21^{e}$ siècles, plus rarement aux auvres du $16^{e}, 17^{e}$ et $18^{e}$ siècle, que dire des textes plus anciens, ceux du Moyen Âge? Les recensions montrent qu'ils sont totalement ignorés. Notre article voudrait défendre l'intérêt d'un enseignement de la littérature médiévale en FLE et soutenir les enseignant.e.s en leur fournissant des pistes d'activités efficaces permettant aux apprenant.e.s de dépasser les difficultés que suppose une lecture littéraire authentique. Pour ce faire, nous inscrivons notre démarche dans le sillage des méthodologies de l'approche globale des textes écrits (Moirand, 1979), et de la lecture interactive (Cicurel, 1991).

Avec l'apparition des approches communicative puis actionnelle dans la didactique des langues étrangères, qui prônent l'utilisation de documents authentiques, la littérature a fait son retour dans les classes de français langue étrangère. Doublement marginalisés au siècle dernier par les méthodes audio orale (MAO) et structuro-globale audiovisuelle (SGAV) pour leurs formes linguistiques trop savantes mais aussi pour leurs contenus culturels trop spécifiques, ${ }^{1}$ les textes littéraires connaissent aujourd'hui un regain d'intérêt. En effet, les discours théoriques leur font désormais la part belle dans l'enseignement du Français langue étrangère (FLE) ${ }^{2}$. Pourtant, il n'en va pas toujours de même dans la pratique. La littérature continue d'angoisser voire de décourager enseignant·e.s et élèves. Si le corpus traditionnellement utilisé dans l'enseignement secondaire et supérieur ${ }^{3}$ emprunte le plus souvent à la littérature des $19^{\mathrm{e}}, 20^{\mathrm{e}}$ et $21^{\mathrm{e}}$ siècles, plus rarement aux œuvres du $16^{\mathrm{e}}, 17^{\mathrm{e}}$ et $18^{\mathrm{e}}$ siècle (Ronsard, Du Bellay, Molière, La Fontaine et Voltaire en sont les principaux représentants), que dire des textes plus anciens, ceux du Moyen Âge ? Le constat est sans appel : ils semblent totalement ignorés. Leur apparente inaccessibilité explique-t-elle qu'on les boude alors même qu'ils représentent avec tout autant de prestige le patrimoine littéraire et culturel français?

Nous souhaitons défendre ici l'intérêt d'un enseignement de la littérature médiévale en français langue étrangère. Notre propos voudrait soutenir les enseignant.e.s en leur fournissant des pistes d'activités efficaces permettant aux apprenant.e.s de dépasser les difficultés linguistiques et culturelles que suppose une lecture littéraire authentique. ${ }^{4}$ Pour ce faire, nous inscrivons notre démarche dans le sillage des méthodologies de l'approche globale des textes écrits, et de la lecture interactive. Dès les années 1980, l'approche globale de Moirand (1979) a remis en question le déchiffrement linéaire du texte et a favorisé la prise en compte du contexte. Dans les années 1990, la lecture interactive de Cicurel (1990) a, quant à elle, transposé les théories de la lecture dans le domaine de l'enseignement de la littérature en FLE. ${ }^{5}$ Les stratégies qui en découlent offrent

1 Notons qu’à l'époque où sont développées ces méthodes, les supports littéraires n'avaient pas forcément disparu des salles de classe pour diverses raisons (prestige de la littérature française, littérature comme symbole de la culture française, traditions d'enseignement, etc.). Il n'en demeure pas moins que ces méthodes bannissent volontairement la littérature de leurs manuels et de leurs formations (voir Puren 1988, p. 208 et suivantes).

2 Nous nous restreignons à mentionner quelques ouvrages généraux au sein d'une bibliographie foisonnante d'articles et de numéros de revue consacrés à la question : voir Fiévet (2013); Defays et al. (2014); Godard (2015); Riquois (2019).

3 Voir par exemple les recensions de Weller (2000) et Maillard-De la Corte Gomez (2009). Le contexte suisse, dans lequel s'inscrit cet article, présente exactement les mêmes lacunes. La consultation des plans d'études cantonaux pour l'enseignement gymnasial du FLE révèle l'absence totale des textes médiévaux, une présence rare de la littérature des $16^{\mathrm{e}}, 17^{\mathrm{e}}$ et $18^{\mathrm{e}}$ siècles et la prédominance des œuvres des $19^{\mathrm{e}}, 20^{\mathrm{e}}$ et $21^{\mathrm{e}}$ siècles. Plans d'études consultés : BS (2018); BL (2014); AG (2016); SO (2019) ; VD (2019) ; FR (2014) ; ZH (2012) ; BE (2017) ; SG (2019) ; TI (2018).

4 Cette lecture authentique s'entend par opposition aux adaptations et aux simplifications des textes littéraires dans la perspective de les rendre accessibles à des apprenant·e-s en langue étrangère d'un niveau donné.

5 Voir Moirand (1979) et Cicurel (1991). Ces méthodologies et les travaux qui les ont complétés marquent aujourd'hui encore la didactique de la littérature en FLE. 
aux apprenant.e.s la possibilité de développer des compétences non linguistiques, transposables dans n'importe quelle langue étrangère. Celles-ci ne se focalisent donc ni sur les obstacles lexicaux ou grammaticaux, ni sur la compréhension détaillée du texte. Elles font appel à des connaissances et des compétences d'un autre ordre, qui visent à rendre les élèves actives et actifs : des savoirs (des connaissances générales, la reconnaissance d'éléments formels et génériques) combinés à des savoir-faire (la capacité de raisonnement, d'anticipation, d'inférence). L'apprenant·e est ainsi soutenu par des stratégies concrètes qui lui permettent de comprendre le texte littéraire au-delà des difficultés linguistiques et culturelles. Dans la perspective qui est la nôtre, il s'agira d'adapter ces méthodologies à des textes narratifs plus anciens, et de fait habituellement absents des classes de FLE.

Dans un premier temps, nous justifierons notre démarche en nous référant au discours théorique actuel sur l'enseignement de la littérature en langue étrangère. Dans un second temps, nous montrerons dans quelle mesure les acquis de la didactique de la lecture en usage dans l'enseignement du FLE peuvent s'appliquer à la littérature médiévale. Pour ce faire, nous nous intéresserons d'abord à la perception globale du texte, puis à « l'encyclopédie » de la lectrice ou du lecteur-apprenant, enfin, aux stratégies d'anticipation des étapes d'un récit.

\section{Justification de la démarche}

La parution en 2001 du Cadre européen commun de référence pour les langues (CECRL) et de ses descripteurs des niveaux de compétence en langue étrangère a renouvelé le discours didactique. Rappelons que l'approche communicative avait déjà proposé de compléter les savoirs linguistiques traditionnels par les savoir-faire et les savoir-être socioculturels. Avec le CECRL, l'approche actionnelle et son concept de tâche viennent enrichir cette distinction. En outre, le développement des approches plurielles favorise l'acquisition de connaissances et de compétences interculturelles et plurilingues. Depuis 2017, l'élément complémentaire "Littérature » du Portfolio européen des langues (Hodel, 2017) propose désormais des descripteurs permettant d'évaluer à la fois les compétences langagières et les compétences en lecture de textes littéraires. Enfin, le Conseil de l'Europe a publié en 2018 un Volume complémentaire contenant encore de nouveaux descripteurs consacrés à la lecture littéraire. Notons, avec Godard (2015), que la littérature comme objet et comme discipline s'est également vue transformée :

D’une part, de plus en plus la littérature s'appréhende dans un continuum de pratiques culturelles. D’autre part, la reconnaissance des littératures francophones a modifié la notion même de canon littéraire qui avait jusque-là valeur patrimoniale et nationale, changeant aussi, dans le même mouvement, la représentation des relations entre langue et littérature françaises. [...] la littérature reste ainsi un enjeu fort des politiques éducatives françaises et européennes, à l'articulation du linguistique et du culturel, orienté vers l'expérience de l'altérité. (p. 5-6)

Ces nouveautés contribuent aux réflexions didactiques actuelles sur ce domaine. On le sait, celles-ci alimentent toujours le débat sur le concept de «lecture littéraire », entendu non seulement comme la lecture de la littérature, mais aussi comme "manière de lire ». Selon Dufays (2002), la première conception de la " lecture littéraire " considère le texte « indépendamment de sa réception, il reste un monument déjà saturé de sens et de valeur qu'il s'agit de révéler par une lecture qui s’applique à en célébrer les virtualités ", alors que la seconde vise à "investir des valeurs littéraires dans la lecture elle-même " à travers "trois modélisations distinctes [...] la distanciation, la participation psychoaffective ou le va-et-vient dialectique » (p. 3). En effet, on distingue bien deux modes de lecture, révélant un double mouvement d'adhésion et de distanciation 6 : le premier sollicite la sensibilité, l'imagination, l'immersion, alors que le second rationalise le texte par la construction du sens, l'interprétation symbolique et l'évaluation esthétique ou éthique. Les travaux de Picard (1986) ${ }^{7}$ et Jouve (1993) ont montré la nécessité d'un va-et-vient entre ces deux modes de lecture, l'intégration d'une lecture savante et d'une lecture authentique au sein d'une même activité, ainsi que la prise en compte des tensions qui en découlent :

6 Toutes choses étant égales, on relève chez la plupart des théoricien·ne·s une conception dichotomique de la lecture qu'il faudrait évidemment replacer dans son contexte épistémologique mais qu'il est intéressant de rappeler : Riffaterre (1982) oppose "lecture heuristique » et lecture " herméneutique "; Stierle (1979) « lecture quasi-pragmatique » et "lecture pseudoréférentielle "; Eco (1985) "l'utilisation du texte " et la " coopération interprétative "; Gervais (1993) la " régie de la progression " et la "régie de la compréhension".

7 Rappelons que chez Picard (1986), le lecteur est à la fois «liseur " (instance physique), "lu " (instance psychoaffective) et " lectant " (instance intellectuelle) et que la lecture est considérée comme littéraire lorsqu'il y a mise en tension entre certaines valeurs $\mathrm{du}$ « lu » et $\mathrm{du}$ « lectant » (par exemple, sens/signification, réalité/fiction, conformité/subversion). 
[...] le rapport entre l'ancrage et le désancrage du sens, la fonction référentielle et la fonction poétique, les rapports passionnel et rationnel, la subjectivité et l'intersubjectivité, la centration sur un corpus restreint et l'accueil d'un corpus ouvert, le privilège accordé aux valeurs " modernes » et celui accordé aux valeurs " classiques» $[. .$.$] . (Dufays, 2002, p. 5-6)$

Cette conception de la " lecture littéraire " favorise à la fois l'émergence d'un "sujet lecteur " (Langlade et Rouxel, 2005) conscient de sa propre subjectivité et de son rôle actif dans la lecture, mais aussi la mise en place d'une approche collaborative. Les apprenant.e.s sont en effet encouragé.e.s à confronter leurs hypothèses et leurs interprétations, en vue de soutenir la compréhension du texte et de renforcer les stratégies de lecture des élèves moins aguerri.e.s.

Cette contextualisation théorique est fondamentale pour saisir l'intérêt de notre démarche dans le cadre d'un enseignement favorisant l'utilisation de toutes les ressources de la pédagogie moderne. Cette démarche souscrit en effet aux principes qui régissent la didactique actuelle : elle vise à rendre les apprenant.e.s actives et actifs 8 et conscient.e.s de leur rôle dans l'accès à la compétence lectorale et de manière générale dans l'apprentissage de la langue et de la culture française ; elle propose l'utilisation de documents authentiques ; elle participe au développement de compétences interculturelles et plurilingues en proposant des textes, en ancien français, d'une époque éloignée ; elle adopte une méthodologie qui allie lecture savante et lecture authentique, distanciation et adhésion ; pour finir, elle se prête particulièrement bien à la pédagogie de projet et à une approche collaborative.

\section{Pour une perception globale des textes}

Notre démarche souhaite ouvrir l'accès au texte médiéval dans sa version originale. En effet, on sait que toute traduction entraine une déperdition du sens et des idiomatismes, un appauvrissement du style et de la musicalité, ainsi qu'une trahison des images. Cependant, il est difficile de se passer d'une édition bilingue en classe de FLE. Celle-ci présente d'ailleurs l'avantage d'ouvrir une perspective historique et plurilingue en faisant apparaitre deux états d'une même langue. Les apprenant.e.s sont ainsi amené.e.s à réfléchir sur le fonctionnement et l'évolution de la langue et à adopter un regard métalinguistique. De plus, le recours à l'édition bilingue n'interdit pas de proposer une lecture qui prendrait prioritairement appui sur le texte en ancien français, notamment dans le cas des activités de repérage chères à l'approche globale. Celle-ci encourage à la perception globale des mots et des phrases en se fondant sur l'architecture du texte, les savoirs-extralinguistiques, ainsi que les intentions de lecture. Un premier temps d'observation (la présentation du texte, son support, le paratexte, etc.) amène la lectrice ou le lecteur-apprenant à reconnaitre l'émetteur, le récepteur et le genre. Puis un second temps s'appuie sur des stratégies de repérage permettant d'amorcer la compréhension : les questions qui ?, quoi ?, quand ?, où ?, mais aussi l'examen des articulateurs (logiques, rhétoriques, déictiques, spatio-temporels), des répétitions ou des parasynonymes d'un terme, la prise en compte du contexte de production, ou encore la mise en relation de plusieurs parties du texte, soutiennent l'apprenant.e dans la construction du sens.

La littérature narrative médiévale s'adapte particulièrement bien à de telles activités, notamment lorsqu'il s'agit de familiariser les apprenant.e.s à des types et des genres textuels, ou à différents niveaux de stéréotypie (clichés, lieux communs, topö̈, fonctions, idéologèmes) ${ }^{9}$. On pourra par exemple travailler sur le prologue du roman et ses différents topoï qui sont aisément repérables (l'intention didactique, la dédicace à un destinataire prestigieux, la vertu salutaire du travail ou le choix du sujet pour en citer quelques-uns) ; ou encore sur le procédé de l'entrelacement - ce que l'on appelle aujourd'hui " les récits enchassés » -, identifiable grâce à des formules récurrentes ("Or dist li contes») ; sur le style formulaire des chansons de geste et particulièrement sur les syntagmes figés (Roland est preux et Olivier est sage, Guillaume a le " curt ne s ») et sur la répétition de scènes (les plaines sont toujours grandes et désertes, le chevalier descend toujours de cheval sous un pin) ; ou enfin, pour les textes plus tardifs des $15^{\mathrm{e}}$ et $16^{\mathrm{e}}$ siècles, sur le système de repérage textuel ${ }^{10}$ formé par les rubriques - autrement dit les premiers titres de chapitre souvent très longs et descriptifs à cette époque -, le

8 Dans sa méthodologie, sur laquelle nous fondons notre démarche, Cicurel parle d'une " lecture active, sorte de lecturerecherche où le lecteur fait des hypothèses sur le sens à partir de certains éléments (titres, mises en page, légendes) et de parcours sur l'espace du texte pour reconnaitre des éléments utiles à l'acquisition de la signification» (1991, p. 14).

9 Voir les travaux de Dufays sur le concept de stéréotype (1991, 1994, 2001 ; Dufays et Gemenne, 2010; Dufays et Kervyn, 2015).

10 Sur le développement du système de repérage textuel dans la littérature narrative, nous renvoyons à Hasenohr (1990, p. 273-287). 
découpage en paragraphes, mais aussi l'illustration (enluminures ou gravures) qui seront d'une grande aide pour la compréhension du contenu de l'œuvre et de sa structure. Rappelons que la littérature médiévale, du fait de ses conditions de production et de réception - en particulier son oralisation -, repose sur une esthétique de la répétition qu'il serait dommage de ne pas exploiter dans la perspective d'un enseignement du français langue étrangère. On se souvient de méthodes didactiques pour l'apprentissage des langues, mettant avant tout l'accent sur une pratique orale, qui s'appuient sur la répétition de structures clés. ${ }^{11}$ Dufays (2015) rappelle également que la stéréotypie en littérature, qui caractérise particulièrement les textes médiévaux, constitue pour les apprenant.e.s " un matériau verbal et sémantique manipulable et transformable de diverses manières; [elle est] donc un excellent support pour la créativité et l'apprentissage de la maitrise technique du langage » (p. 260).

Le développement d'une perception globale du texte à travers ces activités de repérage sera également l'occasion d'aborder la compétence plurilingue. Des exercices qui en favorisent l'acquisition complèteront ce travail centré sur des acquis non linguistiques. La mise en parallèle du texte original, soit avec d'autres langues connues des apprenant.e.s, soit avec le français moderne, par conséquent dans une perspective à la fois inter- et intralinguale, présente des aspects ludiques qui ne manqueront pas de susciter la motivation. Des comparaisons des structures morphosyntaxiques (comparer la déclinaison du substantif, la syntaxe, la formation des temps, etc.), des dérivations ou des évolutions morphologiques à partir de mots de la même famille, des confrontations d'expressions, de proverbes, de colocations, en somme toutes les activités typiques des approches plurielles des langues et des cultures, ${ }^{12}$ s'adapteront parfaitement à un enseignement de la lecture du texte médiéval. On pourra également proposer des tâches sur la prononciation de l'ancien français et la phonétique/phonologie afin de poursuivre la réflexion métalinguistique des apprenant.e-s sur l'évolution de la langue. Notre expérience d'enseignante de littérature médiévale pour un public germanophone à l'université a souvent révélé de meilleurs résultats chez des étudiant.e.s germanophones que francophones dans la compréhension et la traduction de l'ancien français vers le français moderne. Les francophones ont en effet davantage de peine à se détacher des structures syntaxiques et morphologiques de leur langue maternelle dans son état moderne. Ainsi, des activités ponctuelles autour du plurilinguisme soutiendront, à l'occasion, la compréhension linguistique du texte ancien tout en participant de manière inédite au développement de la compétence plurilingue. Mais l'approche du texte médiéval ou renaissant, pour rester réaliste et ne pas décourager l'apprenant.e, se veut avant tout globale.

\section{3. Élargir "l'encyclopédie " des Lectrices ou lecteurs- apprenants}

Comme nous l'avons évoqué en introduction, la méthodologie de la lecture interactive se fonde sur les théories de la lecture, en particulier les travaux d'Umberto Eco (1985). L'auteur développe les concepts bien connus de " coopération " entre le lecteur ou la lectrice et le texte et d'" encyclopédie " du lecteur ou de la lectrice, qui rassemble à la fois des connaissances référentielles (des expériences culturelles et intellectuelles accumulées tout au long de la vie sur un thème donné) et des connaissances sur le fonctionnement des textes acquises dans le cadre d'un apprentissage scolaire ou grâce à des lectures antérieures (on parle alors de compétence intertextuelle). Des schémas de contenu (des épisodes situationnels) et des schémas formels (des suites conventionnelles d'actions) viennent ainsi alimenter cette " encyclopédie ». Le lecteur ou la lectrice, en lisant, opère un va-etvient permanent entre le texte et ce répertoire d'informations qui lui est propre. Il ou elle émet des hypothèses à partir de scénarios communs et intertextuels qui seront infirmées au fur et à mesure de sa lecture - Eco évoque des "promenades inférentielles"-, il ou elle anticipe donc les actions, le genre ou la valeur pragmatique du texte. Par analogie avec ce qu'il ou elle connait déjà, il ou elle est capable de construire du sens et d'avancer malgré les obstacles linguistiques ou culturels. Ces automatismes sont ceux que tout.e lecteur ou lectrice met naturellement en place lorsqu'il ou elle lit en langue maternelle. La didactique de la lecture en langue étrangère considère qu'ils peuvent finalement être transposés dans n'importe quelle langue, à la condition d'un lâcher prise par rapport à une lecture mot à mot généralement laborieuse et entrainant le blocage des compétences non linguistiques dont nous avons parlé. Cicurel (1991) rappelle la distinction entre les unités de rang inférieur (connaissance du code : graphie, mots, phrases) et les unités de rang supérieur (la structuration sémantique, la connaissance du contenu référentiel, la reconnaissance du genre textuel) : "Paradoxalement les observations

11 On pense évidemment aux méthodes audio orale (MAO) et structuro-globale audiovisuelle (SGAV) ou, plus récemment, à l'approche neurolinguistique pour l'appropriation des langues (ANL). Voir à ce propos les travaux de Germain et Netten.

12 En particulier les approches de l'intercompréhension entre langues parentes et l'éveil aux langues (voir Candelier, 2012). 
faites sur les lecteurs en langue étrangère ont montré qu'il y a comme un abandon des indices de niveau supérieur alors que précisément le lecteur en aurait davantage besoin qu’en langue maternelle pour faciliter sa compréhension" (p. 12). Elle ajoute que :

La carence de compétence linguistique qui oblige le lecteur à un déchiffrement fastidieux des unités de rang inférieur entraine un blocage de la perception des unités de rang supérieur - la structure textuelle, les schémas de contenu, les connaissances pragmatiques liées au texte qu'il pourrait éventuellement retrouver même sans comprendre l'intégralité du code. (p. 12)

Ainsi, des activités de pré-lecture sont proposées, dont l'objectif est d'élargir l' " encyclopédie " des lectrices ou lecteurs-apprenants afin de les rendre capables par la suite de donner du sens au texte (Cicurel, 1991, p. 125-153). Outre la mise à disposition des informations habituelles sur l'époque, le genre, le contexte ou encore l'auteur ou l'autrice, Cicurel (1991) suggère de sensibiliser les élèves aux registres littéraires en recourant à la comparaison avec des textes non littéraires. La description et le portrait de presse pourront ainsi mettre en exergue les spécificités de ces types de discours au sein de la littérature, tandis que le fait-divers pourra, quant à lui, préparer les apprenant.e.s au repérage de la trame événementielle d'un récit.

Là encore, la littérature narrative médiévale se prête particulièrement à ces stratégies. Les difficultés linguistiques qu'elle présente peuvent être encore une fois compensées par le recours à son caractère stéréotypé. La stéréotypie œuvre à la fois au plan du récit, mais aussi au plan discursif. ${ }^{13}$ Le texte se présente comme un enchainement de thèmes : pour le roman courtois, par exemple, la quête chevaleresque ou la conquête de la dame ; pour la chanson de geste, les préliminaires à la guerre ou la bataille. Ces thèmes sont eux-mêmes constitués de motifs qui présentent à leur tour des formules plus ou moins stéréotypées. On pourra ainsi travailler sur des motifs littéraires en fonction du genre abordé : pour le roman courtois, par exemple, le portrait de la dame, la description des combats chevaleresques ou les scènes d'adoubement ; pour la chanson de geste le planctus - longue plainte d'un ou plusieurs personnages, généralement en situation de deuil -, la prière du plus grand péril - un chevalier en péril invoque l'aide de Dieu -, ou les fameuses listes de noms. Tous ces exemples possèdent chacun un style et une structure bien codifiés, comme nous le montrons ci-dessous avec la prière du plus grand péril, typique de la chanson de geste (Figure 1). Ces motifs sont identifiables autant par leur répétition (à l'intérieur d'une même œuvre ou à travers des œuvres diverses) que par leur forme. Une connaissance même parcellaire de leur architecture discursive permettra ainsi aux apprenant·e•s de les repérer dans le texte.

\section{Tableau 1}

Structure de la prière du plus grand péril (La Prise d'Orange, 2010)

\begin{tabular}{|l|l|}
\hline \multicolumn{2}{|c|}{ Prière du plus grand péril dans La Prise d'Orange } \\
\hline Glorïeus Sire, qui formas tote gent & 1. Apostrophe à Dieu \\
Et de la Virge fus nez en Belleant & 2. Énumération des prodiges divins \\
Quant li troi roi vos aloient querant & \\
Et en la croiz vos penerent tirant, & \\
Et de la lance fus feruz enz el flanc & \\
Ce fist Longis qui estoit non veant, & \\
Que sanc et eve l'en vint as poinz corant, & \\
Tert a ses eulz, si ot alumement- & 3. Acte de foi avec formule récurrente \\
Si com c'est voirs que ge vois devisant, & 4. Requête \\
Gardez noz cors de mort et de torment, & \\
Ne nos ocient Sarrazin et Persant. (v. 498-505) &
\end{tabular}

\section{Anticiper les étapes d'un récit}

La didactique de la lecture a également montré l'intérêt d'un travail sur les constituants narratifs. ${ }^{14}$ Cette démarche permet en effet de mettre à disposition des apprenant.e.s de véritables canevas d'histoires dans la perspective, toujours, de soutenir la compréhension. Cicurel (1991) conseille d'organiser des activités autour de différents blocs narratifs qui constituent autant de repères pour la lectrice ou le lecteur-apprenant et l'amènent

\footnotetext{
13 Voir entre autres Zumthor (1972).

14 Mentionnons Riquois (2009), qui utilise une démarche similaire dans sa thèse.
} 
à interroger le texte. Elle propose l'utilisation du schéma traditionnel de la séquence narrative développé par la linguistique structurale : 1 . état initial ; 2 . complication ; 3. péripéties ; 4. résolution ; 5 . dénouement (p. 145). Caré et Debyser (1991) utilisent, quant à eux, un schéma beaucoup plus détaillé s'inspirant de l'analyse structurale du conte merveilleux proposée par Propp (1965) qui a influencé, on le sait, les théories de Barthes, Greimas et Todorov sur la structure du récit. Ce schéma se révèlera très utile dans le cadre de la lecture d'un récit merveilleux (comme les romans de Chrétien de Troyes ou les Lais de Marie de France), qui reproduit une structure narrative proche de celle du conte. On pourra le vérifier en observant la trame narrative des aventures de Perceval dans Le Conte du graal de Chrétien de Troyes (Figure 2).

\section{Tableau 2}

Trame narrative des aventures de Perceval dans Le Conte du graal de Chrétien de Troyes à partir du canevas proposé par Caré et Debyser (p. 148-149).

\begin{tabular}{|c|c|}
\hline $\begin{array}{l}\text { Canevas proposé par Caré et Debyser sur le modèle de } \\
\text { l'analyse structurale du conte par Propp }\end{array}$ & $\begin{array}{l}\text { Constituants narratifs des aventures de Perceval dans Le Conte } \\
\text { du graal de Chrétien de Troyes }\end{array}$ \\
\hline 1. Choix d'un héros. & $\begin{array}{l}\text { 1. Perceval, jeune garçon naïf et sans éducation, surprotégé } \\
\text { par sa mère. }\end{array}$ \\
\hline $\begin{array}{l}\text { 2. Il manque à ce héros quelque chose pour être heureux (un } \\
\text { objet, l'amour, la richesse, etc.). }\end{array}$ & $\begin{array}{l}\text { 2. Il est en quête de son identité. Sa mère la lui a cachée pour } \\
\text { le protéger (il est issu d'une grande famille de chevaliers } \\
\text { tous morts au combat). }\end{array}$ \\
\hline 3. Quelqu'un le renseigne ou le conseille. & $\begin{array}{l}\text { 3. Il rencontre des chevaliers dans la forêt et veut devenir } \\
\text { comme eux. }\end{array}$ \\
\hline 4. Le héros part à l'aventure. & $\begin{array}{l}\text { 4. Il part se faire adouber à la cour du roi Arthur. Plusieurs } \\
\text { aventures se succèdent. }\end{array}$ \\
\hline 5. En chemin, il rencontre un ami ou un allié. & $\begin{array}{l}\text { 5. Gornemant de Goort s'occupe de son éducation et en fait } \\
\text { un vrai chevalier. }\end{array}$ \\
\hline $\begin{array}{l}\text { 6. Seul, ou avec l'aide de cet allié, il rencontre divers } \\
\text { obstacles qu'il surmonte. }\end{array}$ & $\begin{array}{l}\text { 6. Il démontre de grandes qualités en tant que chevalier au } \\
\text { cours de nouvelles aventures. }\end{array}$ \\
\hline 7. Il parvient à l'endroit où se trouve ce qu'il cherche. & 7. Il arrive au château du graal. \\
\hline $\begin{array}{l}\text { 8. Un puissant ennemi s'oppose à lui (ogre, roi, dragon, } \\
\text { sorcier, etc.). }\end{array}$ & $\begin{array}{l}\text { 8. Son ennemi est son propre silence devant le cortège du } \\
\text { graal et ses enchantements. }\end{array}$ \\
\hline $\begin{array}{l}\text { 9. Le héros affronte une première fois son ennemi, mais il est } \\
\text { vaincu par lui (blessé, empoisonné, ensorcelé, etc.). }\end{array}$ & $\begin{array}{l}\text { 9. Perceval aurait dû poser des questions mais la faute qu'il } \\
\text { porte en lui (l'abandon de sa mère, suivi de sa mort) le fait } \\
\text { échouer, le château disparait. }\end{array}$ \\
\hline $\begin{array}{l}\text { 10. L'ami (ou un autre ami) du héros vient à son aide (le } \\
\text { libère, le guérit, lui donne un conseil ou une arme, etc.). }\end{array}$ & $\begin{array}{l}\text { 10. Après des années d'errance pour retrouver le château du } \\
\text { graal, il rencontre un ermite qui lui fait des révélations sur } \\
\text { sa famille et l'exhorte à expier ses fautes pour enfin mériter } \\
\text { le graal. }\end{array}$ \\
\hline $\begin{array}{l}\text { 11. Le héros affronte une deuxième fois son ennemi et gagne } \\
\text { (le blesse, le tue, lui prend ce qu'il est venu chercher, etc.). }\end{array}$ & $\begin{array}{l}\text { 11. Le roman reste inachevé mais on imagine aisément la fin } \\
\text { (les continuateurs sont allés dans le même sens). }\end{array}$ \\
\hline $\begin{array}{l}\text { 12. Durant son retour, le héros est poursuivi par des alliés ou } \\
\text { serviteurs de son ennemi (frères, soldats, monstres, etc.). }\end{array}$ & \\
\hline $\begin{array}{l}\text { 13. Il doit les combattre et surmonter divers obstacles, pièges } \\
\text { ou difficultés. }\end{array}$ & \\
\hline 14. Le héros rentre chez lui, dénouement (mariage, etc.). & \\
\hline
\end{tabular}

Il sera particulièrement intéressant de prendre en compte la dimension subjective de la lecture dans le travail sur les constituants narratifs. En effet, les activités qui prendront appui sur un canevas d'histoire seront l'occasion de laisser la place à l'initiative et à l'autonomie des apprenant.e.s. On verra probablement autant de propositions structurelles que de lectrices et lecteurs, selon les représentations et les interprétations subjectives de chacun·e. Par exemple, l'étape 7 ("Il parvient à l'endroit où se trouve ce qu'il cherche ») pourra constituer, en fonction de l'élève, l'arrivée au château d'Arthur, l'arrivée au château du graal ou encore la rencontre avec l'ermite. Les schémas narratifs relatifs à d'autres genres que le roman courtois peuvent également être exploités. Les récits idylliques ${ }^{15}$ sont caractérisés par une trame narrative stéréotypée et reconnaissable : 1 . deux jeunes amoureux

15 Voir Vuagnoux-Uhlig (2009). 
sont séparés par leurs parents afin d'éviter une mésalliance ; 2 . commence une quête pour retrouver l'être aimé avec de nombreuses péripéties qui sont autant d'obstacles permettant d'éprouver leur amour ; 3. le dénouement permet aux amants de se retrouver et de s'unir par le mariage. Ces textes présentent de plus des motifs récurrents qui, après une exploitation préalable, serviront de repères utiles à la compréhension de l'œuvre : la fausse mort, le déguisement des amants, le rendez-vous dans le verger, le don de l'anneau, etc. Dans un registre satirique et comique, aussi bien pour le genre narratif que théâtral, le fabliau, la fable ou encore la farce, lorsqu'ils mettent en scène une supercherie, fonctionnent souvent sur un schéma basique : 1. convoitise d'un personnage par rapport à un autre (argent, nourriture, épouse, etc.) ; 2. mise en place du piège ; 3. succès de la démarche ; 4 . morale ou retournement de situation. Notons que ce schéma est évidemment très réducteur et ne constitue pas une caractéristique structurelle de ces différents genres. Ménard (1983) note très justement que "la pluralité des thèmes entraine immanquablement la diversité des structures " (p. 37). Néanmoins, ce canevas pourra se révéler particulièrement utile dans notre contexte pédagogique. On pourra également aborder des œuvres comme Le Roman de Renart ou La farce de Maitre Pathelin à partir de ce schéma et ouvrir ainsi des portes vers la compréhension et le sens. Par ailleurs, ces textes ont le mérite de susciter un certain intérêt auprès des apprenant.e.s : outre le registre comique commun à toute cette littérature satirique, l'anthropomorphisme du Roman de Renart ou des fables en général, souvent rattaché dans les représentations actuelles au monde de l'enfance, peut sembler plus accessible aux yeux des lecteurs en FLE.

Ainsi, la connaissance de ces structures narratives permettra à la lectrice ou au lecteur-apprenant confronté à un extrait de littérature médiévale de suivre une logique d'anticipation. Il sera amené à formuler des hypothèses au fil de sa lecture, qui seront éventuellement corrigées. D’autres activités pédagogiques devront évidemment encadrer cette seconde phase de lecture. Elles pourront s'inscrire dans l'une des trois interactions proposées par Cicurel (1991, p. 134). La première interaction s'opère du lecteur ou de la lectrice vers le texte : le lecteur ou la lectrice émet des hypothèses de lecture et construit sa compréhension de l'œuvre à partir de questions ouvertes, de Q.C.M., de résumés lacunaires, etc. La deuxième interaction s'effectue en sens inverse, du texte vers le lecteur ou la lectrice : il ou elle livre, à partir de questions à visée subjective ${ }^{16}$ ou du travail sur les constituants narratifs dont nous avons parlé, ses impressions de lecture, son interprétation personnelle et décrit l'effet que le texte produit sur lui ou elle. C'est l'occasion de contextualiser ou d'actualiser le texte, de l'interpréter symboliquement, de l'évaluer esthétiquement et éthiquement en prenant en compte à la fois la lecture authentique et la lecture analytique, toujours dans cette dynamique de va-et-vient chère à Picard et Jouve. La troisième interaction met en relation les apprenant.e.s eux et elles-mêmes : les élèves rassemblent leurs hypothèses mais aussi leurs ressentis, ils et elles confrontent leurs différentes subjectivités afin de construire un point de vue global.

Pour finir, notons que les activités de repérage textuel, le travail sur les motifs littéraires et l'analyse de structures narratives pourront être réinvestis dans la phase d'après-lecture, notamment lors des tâches de production écrite " classiques" (activités de transformation, d'imitation, d'amplification, de transposition ou de traduction). Celles-ci viendront à leur tour renforcer une nouvelle fois la compétence lectorale. En effet, si la relation entre les compétences de lecture et d'écriture n'est plus à démontrer, on oublie souvent qu'elle est à double sens. Certes, la production écrite succède souvent aux activités de lecture, néanmoins, pour faire progresser les apprenant.e.s en lecture, il faut aussi les faire écrire. Ces activités devront également leur permettre de voir leur statut de «sujet lecteur " évoluer vers celui de "sujet scripteur » et d'affirmer leur légitimé à s’inscrire dans une démarche littéraire. Pour garantir le succès de cette transformation, il est impératif de ne pas traiter les tâches de manière isolée mais de les rattacher à des objectifs initiaux et à une progression didactique. Dans le même temps, il sera également nécessaire de prendre du recul par rapport au mode de lecture adopté dans les deux premières phases de travail. Celui-ci insiste en effet sur les caractéristiques thématiques et formelles des récits, au risque de conditionner l'apprenant·e-scripteur. Il faudra alors préserver la liberté créative en évitant de survaloriser la conformité à la consigne, tout en prônant l'écart maitrisé. Godard (2015) invite ainsi à penser l'écriture créative de la même manière que la lecture authentique :

Enfin, de même que, dans les pratiques réelles, l'on ne lit pas la littérature en se donnant seulement pour objectif de mieux lire, mais aussi pour les émotions qu'elle suscite en nous et pour ce qu'elle nous apporte, de même en classe de FLE, au-delà d'un mieux-écrire et d'un mieux-lire, on peut penser l'écriture créative en redonnant sa place au sujet caché, et parfois nié, dans tout «apprenant »-écrivant. (p. 190)

16 Massol (2017) en donne quelques exemples : «quelle image vous vient à l'esprit ? "; " faites-vous un lien avec un autre texte, un film, une musique? »; " le texte évoque-t-il un souvenir personnel ? »; " quelle appréciation globale portez-vous sur le style, le sens, l'intérêt du texte?" (p. 4). 
Dans une perspective plus moderne et innovante, la lecture des textes médiévaux trouvera sans difficulté sa place au sein d'une pédagogie de projet et d'une approche collaborative. Les macro-tâches spécifiques aux projets s'articulant autour de la lecture d'un roman comme le débat littéraire, la théâtralisation, la création d'une B.D/d'une adaptation cinématographique, l'écriture/la conception collaborative d'un roman/d'une édition/d'une anthologie, devront s'adapter aux particularités du roman médiéval. On pourra par exemple proposer la création d'un fac-similé de manuscrit à partir du texte étudié (mise en page, reproduction des caractères gothiques, des rubriques, des enluminures) qui réinvestira les connaissances acquises dans la phase de perception globale de l'œuvre, par exemple les activités sur le paratexte. Les élèves pourront y insérer des modifications ou une fin nouvelle, à la manière des copistes de l'époque, réemployant ainsi leurs connaissances sur les constituants narratifs. Une autre macro-tâche pourrait se réaliser dans la performance du texte à la façon du jongleur médiéval (oralisation ou mise en musique du texte, intégration de musiques d'époque, insertions dans le texte de marques d'oralité comme les adresses au public) qui réinvestirait cette fois, entre autres, le travail sur les approches plurielles des langues et des cultures, notamment la prononciation, la phonétique et la phonologie de l'ancien français.

\section{Conclusion}

Le terrain des possibilités d'enseignement de la littérature médiévale en classe de FLE est encore long à défricher. Notre présent article vise avant tout à montrer l'intérêt de la démarche, notamment dans la didactique actuelle du FLE, ainsi qu'à fournir des pistes. Ces propositions méritent d'être développées : à la question des activités et de leur mise en œuvre, s'ajoute celle du corpus praticable et donc des critères de sélection des œuvres, ainsi que celle du niveau de compétence du public visé.

Ce qui, pour nous, ne fait aucun doute, c'est la légitimité de la lecture de ces textes auprès d'apprenant.e.s de français langue étrangère. Si l'argument de la valeur patrimoniale et culturelle de cette littérature est avéré, celui de l'attrait de ses thématiques (la chevalerie, le merveilleux, la quête identitaire) pour des apprenant.e·s qui côtoient quotidiennement des univers inspirés du Moyen Âge dans leurs loisirs (lectures, jeux vidéo, cinéma et séries télévisées) est tout simplement indiscutable. On ne peut ainsi qu’encourager vivement le développement d'autres travaux, voire la conception d'une didactique de la littérature médiévale pour la classe de FLE.

\section{Bibliographie}

Candelier, M. (dir.). (2012). Le CARAP : un cadre de référence pour les approches plurielles des langues et des cultures : compétences et ressources. Centre européen pour les langues vivantes, Conseil de l'Europe. https://www.ecml.at/Portals/1/documents/ECMLresources/CARAP-FR.pdf?ver $=2018-03-20-120658-740$

Caré, J.-M., et Debyser, F. (1991). Jeu, langage et créativité. Hachette.

Cicurel, F. (1991). Lectures interactives en langue étrangère. Hachette FLE.

Defays, J.-M, Delbart, A.-R., Hammami, S., et Saenen, F. (dir.). (2014). La Littérature en FLE. État des lieux et nouvelles perspectives. Hachette FLE.

Dufays, J.-L. (1991). Lire avec les stéréotypes. Les conditions de la lecture littéraire en classe de français. Enjeux, 23, 5-18.

Dufays, J.-L. (1994). Stéréotype et lecture. Mardaga.

Dufays, J.-L. (2001). Le stéréotype, un concept clé pour lire, penser et enseigner la littérature. Dans R. Gauthier (dir.), Le stéréotype : usages, formes et stratégies. Actes du $21^{e}$ colloque d'Albi Langages et signification (p. 19-30). CALS/CPST.

Dufays, J.-L. (2002). Les lectures littéraires : évolution et enjeux d'un concept. Tréma, 19, 5-16.

Dufays, J.-L., Gemenne, L., et Ledur, D. (2015). Pour une lecture littéraire : Histoire, théories, pistes pour la classe. De Boeck.

Dufays, J.-L., et Kervyn, B. (2010). Le stéréotype, un objet modélisé pour quels usages didactiques ? Éducation et didactique, 4(1), 53-80.

Eco, U. (1985). Lector in fabula. La coopération interprétative dans les textes narratifs. Grasset.

Fiévet, M. (2013). La littérature en classe de FLE. CLE International.

Gervais, J.-B. (1993). À l'écoute de la lecture. VLB éditeur.

Godard, A. (dir.). (2015). La Littérature dans l'enseignement du FLE. Les Éditions Didier.

Hasenohr, G. (1990). Les systèmes de repérage textuel. Dans H.-J. Martin, et J. Vezin (dir.), Mise en page et mise en texte du livre manuscrit (p. 273-287). Promodis.

Hodel, H.-P. (dir.). (2017). Complément PEL III pour l'enseignement de la littérature au gymnase. Guide pour les enseignants. Schulverlag plus $A G$.

Jouve, V. (1993). La Lecture. Hachette.

Langlade, G., et Rouxel, A. (dir.). (2005). Le Sujet lecteur. Lecture subjective et enseignement de la littérature. PUR.

La Prise d'Orange, chanson de geste (fin 12e-début 13e siècle). (2010). Dans C. Lachet (dir.), v. 498-508. Champion.

Maillard-De la Corte Gomez, N. (2009). Quel corpus de textes littéraires dans les formations de français langue étrangère à l'université ? Discours et pratiques d'enseignants. Le Langage et l'Homme, 44(1), 17-30. 
Massol, J.-F. (2017). Facilités de l'actualisation, difficultés de la contextualisation dans un échange en classe de 1ère L. Recherches et Travaux, 91. http://journals.openedition.org/recherchestravaux/945

Ménard, P. (1983). Les Fabliaux, contes à rire du Moyen Âge. PUF.

Moirand, S. (1979). Situations d'écrit. Compréhension, production en langue étrangère. CLE International.

Picard, M. (1986). La Lecture comme jeu. Minuit.

Propp, V. (1965). Morphologie du conte. Seuil.

Puren, C. (1988). Histoire des méthodologies de l'enseignement des langues. Nathan-CLE International.

Riffaterre, M. (1982). L'illusion référentielle. Dans R. Barthes, L. Bersani, P. Hamon, M. Riffaterre, et I. Watt (dir.), Littérature et réalité (p. 91-118). Seuil.

Riquois, E. (2009). Pour une didactique des littératures en français langue étrangère : du roman légitimé au roman policier. Thèse de doctorat en linguistique, Université de Rouen, France.

Riquois, E. (2019). Lire et comprendre en FLE. Hachette.

Stierle, K. (1979). Réception et fiction. Poétique, 39, 299-320.

Vuagnoux-Uhlig, M. (2009). Le couple en herbe. Galeran de Bretagne et L'Escoufle à la lumière du roman idyllique médiéval. Droz.

Weller, F. R. (2000). Literatur im Französischunterricht heute. Bericht über eine grössere Erhebung zum Lektüre-Kanon. Französisch heute, 2, 138-159.

Zumthor, P. (1972). Essai de poétique médiévale. Seuil.

Mots-clés : Didactique de la littérature ; littérature médiévale ; approche globale ; lecture interactive ; plurilinguisme

\section{Mittelalterliche Literatur im Französischunterricht lesen: eine Utopie ?}

Zusammenfassung

Die Literatur, die traditionell im Rahmen der Sekundar- und Hochschulbildung des Französischen als Fremdsprache verwendet wird, stammt am häufigsten aus der Literatur des 19., 20. und 21. Jahrhunderts und seltener aus den Werken des 16., 17. und 18. Jahrhunderts. Aber was ist mit älteren Texten aus dem Mittelalter? Die Daten zeigen, dass sie bisher völlig ignoriert werden. Unser Artikel möchte das Interesse am Unterrichten mittelalterlicher Literatur im Unterricht „Französisch als Fremdsprache“ wecken und Lehrer dabei unterstützen. Ein Ziel ist, effektive Instruktionen für Lernaktivitäten bereitzustellen, die es den Lernenden ermöglichen, Schwierigkeiten beim Lesen von Originalliteratur zu überwinden. Zu diesem Zweck verwenden wir in unserem Ansatz die Methoden des «approche globale des textes écrits» (Moirand) und der «lecture interactive» (Cicurel).

Schlagworte: Literaturdidaktik; mittelalterliche Literatur; globaler Ansatz; interaktives Lesen; Mehrsprachigkeit

\section{Leggere la letteratura medievale in una classe di francese come lingua seconda: un'utopia?}

Riassunto

Se il corpus letterario tradizionalmente utilizzato nell sistruzione secondaria e superiore del francese come lingua seconda proviene spesso dalla letteratura del XIX, XX e XXI secolo e più raramente dalle opere del XVI, XVII e XVIII secolo, che dire dei testi più antichi del Medioevo? I dati mostrano che vengono completamente ignorati. Il nostro articolo mira a difendere linteresse nell insegnamento della letteratura medievale in francese come lingua straniera e a sostenere gli insegnanti fornendo loro percorsi di attività efficaci che consentano agli studenti di superare le difficoltà della lettura autentica di opere letterarie. Per questo, basiamo il nostro approccio sui metodi dell'approccio globale dei testi scritti (Moirand) e della lettura interattiva (Cicurel).

Parole chiave: Didattica letteraria; letteratura medievale; approccio globale; lettura interattiva; multilinguismo 


\section{Reading medieval literature in French lessons: a utopia ?}

Summary

The literature traditionally used in secondary and higher education of French as a foreign language most often comes from the literature of the 19th, 20th and 21st centuries and less often from the works of the 16th, 17th and 18th centuries. But what about older texts from the Middle Ages? The data shows that they are completely ignored. Our article aims to defend the interest in teaching medieval literature in French as a foreign language and to support teachers. The aim is to provide effective activity tracks that allow learners to overcome the difficulties of authentic literature reading. For this, we base our approach on «global approach to written texts» (Moirand) and «interactive reading» (Cicurel) methodologies.

Keywords: Literature didactics; medieval literature; global approach; interactive reading; multilingualism

Gaëlle Burg, Professeure docteure habilitée en langue et littérature française, G. Burg enseigne depuis 2008 la littérature et le FLE dans le supérieur et le secondaire II (région Bâle). En 2019, elle devient Dozentin à la FHNW (didactique du FLE Sec. II). Ses recherches portent sur la littérature médiévale, l'histoire du livre à la Renaissance et la didactique de la littérature.

Hofackerstrasse 30, CH-4132 Muttenz

E-Mail: Gaelle.burg@fhnw.ch 